\title{
Double whammy
}

New drugs to treat malaria - the most deadly form of which is caused by the parasite Plasmodium falciparum - are required to combat the emergence of resistance to standard therapies such as chloroquine. In a recent article in Nature, Kelly and colleagues report promising results on a newly synthesized dual-action compound designed to help meet this medical need.

During the erythrocytic phase of malaria, large amounts of toxic free haem are released as a by-product of parasitic feeding on the host red blood cell. The parasite protects itself by converting haem to a non-toxic, crystalline form called haemozoin in the digestive vacuole. This process is thought to be the primary target of quinoline antimalarials, such as chloroquine, and remains an attractive target for future therapeutic development as it is essential for parasite survival and involves an immutable, non-parasite-encoded substrate.

However, mutations in the $P$. falciparum chloroquine resistance transporter (CRT) gene, which enable the parasite to sequester quinoline antimalarials away from their site of action, have led to the recent emergence of resistance. Previous studies had indicated that compounds that interact with CRT can resensitize parasites to the effects of quinolines, and so Kelly et al. investigated the possibility of developing compounds that possessed both activities.
To achieve this goal, the authors designed and synthesized compounds consisting of a tricyclic mainframe of an acridone - a protonconducting ionophore with haem-targeting properties - substituted with side chains that promote trapping of the drug in the parasitic digestive vacuole and confer chemosensitization, to reverse drug resistance. One of the compounds, known as T3.5, demonstrated antimalarial efficacy in vivo in two murine models, with no overt toxicity. In vitro, it acted synergistically with four prototypical quinoline derivates against multidrugresistant strains of $P$. falciparum with different geographic and genetic backgrounds. Of note, synergy of T3.5 with quinoline exhibited a unique profile distinct from that of classical quinoline chemosensitizers - for example, by being effective against a broader range of $P$. falciparum strains.

To examine the interaction of T3.5 with CRT, the compound was screened in CRT mutant lines that are commonly used to characterize quinoline chemosensitizers.

In contrast to classical chemosensitizers, T3.5 synergized with quinine against all of the four point mutations tested with equal efficacy.
Overall, although T3.5 alone does not seem to offer greater efficacy than current antimalarial drugs, its unique chemosensitization profile against multidrug-resistant strains of P. falciparum and the potential to target both the malarial parasite and drug resistance with a single molecule make it an attractive candidate for future development. Such a compound might ultimately be used to create powerful combination therapies with established quinolines that could have an important role in efforts to eradicate malaria.

\section{Katie Kingwell}

ORIGINAL RESEARCH PAPER Kelly, J. X. et al. Discovery of dual function acridones as a new antimalarial chemotype. Nature 8 Apr 2009 (doi:10.1038/nature07937) 